\title{
Null Geodesics and Gravitational Lensing in a Nonsingular Spacetime
}

\author{
Shao-Wen Wei, ${ }^{1}$ Yu-Xiao Liu, ${ }^{1}$ and Chun-E Fu ${ }^{2}$ \\ ${ }^{1}$ Institute of Theoretical Physics, Lanzhou University, Lanzhou 730000, China \\ ${ }^{2}$ School of Science, Xian Jiaotong University, Xi'an 710049, China \\ Correspondence should be addressed to Shao-Wen Wei; weishw@lzu.edu.cn
}

Received 25 August 2014; Revised 9 December 2014; Accepted 22 December 2014

Academic Editor: Elias C. Vagenas

Copyright (c) 2015 Shao-Wen Wei et al. This is an open access article distributed under the Creative Commons Attribution License, which permits unrestricted use, distribution, and reproduction in any medium, provided the original work is properly cited. The publication of this article was funded by SCOAP ${ }^{3}$.

\begin{abstract}
The null geodesics and gravitational lensing in a nonsingular spacetime are investigated. According to the nature of the null geodesics, the spacetime is divided into several cases. In the weak deflection limit, we find the influence of the nonsingularity parameter $q$ on the positions and magnifications of the images is negligible. In the strong deflection limit, the coefficients and observables for the gravitational lensing in a nonsingular black hole background and a weakly nonsingular spacetime are obtained. Comparing these results, we find that, in a weakly nonsingular spacetime, the relativistic images have smaller angular position and relative magnification but larger angular separation than those of a nonsingular black hole. These results might offer a way to probe the spacetime nonsingularity parameter and put a bound on it by the astronomical instruments in the near future.
\end{abstract}

\section{Introduction}

The cosmic censorship hypothesis $[1,2]$ says that singularities that arise in the solutions of Einstein's equations are typically hidden within event horizons and therefore cannot be seen from the rest of spacetime. However, in a semiclassical approximation [3], black holes tend to shrink until the central singularities are reached, which will lead to the breakdown of the theory.

Motivated by the idea of the free singularities, there are several ways to obtain black hole spacetime with no singularities at the center. The one presented in $[4,5]$ was inspired by the noncommutative geometry. The points on the classical commutative manifold are replaced by states on a noncommutative algebra, and the point-like objects are replaced by smeared objects. Thus the singularity problem is cured at the terminal stage of black hole evaporation.

Another way is to introduce a de Sitter core to replace the central singularity. The first one constructed in this way is the Bardeen regular black hole [6-9], which was found to have both an event horizon and a Cauchy horizon. Recently, Hayward proposed a nonsingular black hole solution [10] (Poisson and Israel also derived an equivalent solution based on a simple relation between vacuum energy density and curvature $[11,12])$, which is a minimal model satisfying the asymptotically flat and flatness conditions at the center. Its static region is Bardeen-like. In this nonsingular spacetime, a black hole could be generated from an initial vacuum region and then subsequently evaporate to a vacuum region without singularity [10]. This case was extended to the $d+$ 1 dimensional spacetime and some interesting results were obtained [13]. The quasinormal frequency of this nonsingular spacetime has been recently analyzed in [14] with a significant difference from the singular spacetime. In fact, according to the nature of this spacetime, we can divide it into several cases, that is, the nonsingular black hole, the extremal nonsingular black hole, the weakly nonsingular spacetime, the marginally nonsingular spacetime, and the strongly nonsingular spacetime. Other regular black hole solutions [15-22] can be constructed with the introduction of some external form of matter, such as nonlinear magnetic monopole, electrodynamics, or Gaussian sources, which leads to the fact that they are not vacuum solutions of Einstein's equations.

The subject of gravitational lensing by black holes and compact stars has received great attention in the last ten 
years, basically due to the strong evidence of the presence of supermassive black holes at the center of galaxies. The study can be traced back to [23], where the author examined the gravitational lensing when the light passes near the photon sphere of Schwarzschild spacetime. In [24], the authors showed that, in the case of large values of the scalar charge, the lensing characteristics were significantly different. And the result provides preliminary knowledge on the naked singularity lens. This resurrects the study of the gravitational lensing. After modelling the massive dark object at the galactic center as a Schwarzschild black hole lens, it was found that [2527], similar to Darwin's paper, apart from a primary image and a secondary image resulting by small bending of light in a weak gravitational field, there is theoretically an infinite sequence of very demagnified images on both sides of the optical axis. A similar result was also found in [28, 29]. These images were named as the "relativistic images" by Virbhadra and Ellis [25] and that term was extensively used in later work. Based on lens equation [25, 30], Bozza et al. [31-35] developed a semianalytical method to deal with it. This method has been applied to other black holes [36-61]. These results suggest that, through measuring the relativistic images, gravitational lensing could act as a probe to these black holes, as well as a profound verification of alternative theories of gravity in the strong field regime [37-39]. Furthermore, it can also guide us to detect the gravitational waves at proper frequency $[62,63]$. It is also worthwhile to mention that, in [27], the author pointed out that Bozza's semianalytical method gives small percentage difference of the deflection angle, angular position, and angular separation compared to their accurate values, while it gives large percentage difference of magnification and differential time delays among the relativistic images. Thus one must pay great attention to studying the differential time delays among the relativistic images, and we will not consider that case in this paper.

In $[54,55]$, the authors studied the strong gravitational lensing by a regular black hole with noncommutative corrected parameter. The result showed that gravitational lensing in the strong deflection limit could provide a probe to the noncommutative parameter. In this paper, we mainly focus on the exploration of the lensing features in a nonsingular spacetime with the central singularity replaced by a de Sitter core. At first, we study the nature of the spacetime in different range of the nonsingularity parameter $q$. And, according to it, the spacetime is classified into the nonsingular black hole $q / 2 M \in\left(0, Q_{\mathrm{cr} 1}\right)$, the extremal nonsingular black hole $q / 2 M=Q_{\text {crl }}$, the weakly nonsingular spacetime $q / 2 M \in$ $\left(Q_{\mathrm{cr} 1}, Q_{\mathrm{cr} 2}\right)$, the marginally nonsingular spacetime $q / 2 M=$ $Q_{\mathrm{cr} 2}$, and the strongly nonsingular spacetime $q / 2 M \epsilon$ $\left(Q_{\mathrm{cr} 2}, \infty\right)$. Then, under this classification, we study the lensing features in a nonsingular spacetime in both weak and strong deflection limits. The result shows that, in the weak deflection limit, the influence of the nonsingularity parameter $q$ on the lensing is negligible. Compared with it, $q$ has a significant effect in the strong deflection limit, which is very helpful for detecting the nonsingularity of our universe in the future astronomical observations.

The paper is structured as follows. In Section 2, we study the null geodesics and photon sphere for this nonsingular spacetime. In Section 3, the influence of the nonsingularity parameter $q$ on the lensing in the weak and strong deflection limits is investigated, respectively. In Section 4, supposing that the gravitational field of the supermassive black hole at the center of our Milky Way can be described by the nonsingular metric, we estimate the numerical values of the coefficients and observables for gravitational lensing in the strong deflection limit. A brief discussion is given in Section 5.

\section{Null Geodesics and Photon Sphere}

In [10], Hayward suggested that a nonsingular spacetime, as a minimal model, can be described by the metric

$$
d s^{2}=-f(r) d t^{2}+f(r)^{-1} d r^{2}+r^{2}\left(d \theta^{2}+\sin ^{2} \theta d \phi^{2}\right),
$$

where the metric function $f(r)$ reads

$$
f(r)=1-\frac{2 M r^{2}}{r^{3}+2 q^{2} M},
$$

and its behavior is

$$
\begin{gathered}
f(r) \sim 1-\frac{2 M}{r}+\frac{4 q^{2} M^{2}}{r^{4}}+\mathcal{O}\left(\frac{1}{r}\right)^{7} \quad \text { as } r \longrightarrow \infty, \\
f(r) \sim 1-\frac{r^{2}}{q^{2}}+\frac{r^{5}}{2 q^{4} M}+\mathcal{O}(r)^{7} \quad \text { as } r \longrightarrow 0 .
\end{gathered}
$$

It is quite clear that the spacetime described by the above metric is similar to a Schwarzschild spacetime at large distance, while, at small distance, there is an effective cosmological constant, which leads to regularity at $r=0$. The parameter $q$ is a new fundamental constant on the same ground as $\hbar$ and c. In order to keep some degree of generality, we consider $q$ as a free, model-dependent parameter. On the other hand, it is clear that when $q=0$, there will be a spacetime singularity at $r=0$. So, we can name $q$ as a nonsingularity parameter measuring the nonsingularity of a spacetime.

The outer and inner horizons $r_{ \pm}$are determined by $f(r)=$ 0 . And, for the spacetime with large mass, we approximately have $r_{+}=2 M$ and $r_{-}=q$. In order to compute the null geodesics in this nonsingular spacetime, we follow [64]. Here, we only restrict our attention to the equatorial orbits with $\theta=\pi / 2$. The Lagrangian is

$$
2 \mathscr{L}=-f(r) \dot{t}^{2}+\frac{\dot{r}^{2}}{f(r)}+r^{2} \dot{\phi}^{2}
$$

The generalized momentum can be defined from this Lagrangian as $p_{\mu}=\partial \mathscr{L} / \partial \dot{x}^{\mu}=g_{\mu \nu} \dot{x}^{\nu}$ with its components given by

$$
\begin{gathered}
p_{t}=-f(r) \dot{t} \equiv-E=\text { const }, \\
p_{\phi}=r^{2} \dot{\phi} \equiv L=\text { const, } \\
p_{r}=\frac{\dot{r}}{f(r)} .
\end{gathered}
$$


Substituting (5) and (6) into (4), we find that the Lagrangian $\mathscr{L}$ is independent of both $t$ and $\phi$. Thus, we immediately get two integrals of the motion: $p_{t}$ and $p_{\phi}$. Solving (5) and (6), we easily obtain the $t$ motion and $\phi$ motion:

$$
\begin{gathered}
\dot{t}=\frac{E}{f(r)}, \\
\dot{\phi}=\frac{L}{r^{2} \sin ^{2} \theta} .
\end{gathered}
$$

The Hamiltonian is given by

$$
\begin{aligned}
2 \mathscr{H} & =2\left(p_{\mu} \dot{x}^{\mu}-\mathscr{L}\right)=-f(r) \dot{t}^{2}+\frac{\dot{r}^{2}}{f(r)}+r^{2} \dot{\phi}^{2} \\
& =-E \dot{t}+L \dot{\phi}+\frac{\dot{r}^{2}}{f(r)}=\delta .
\end{aligned}
$$

Here $\delta$ is another integral of the motion. And $\delta=-1,0,1$ are for spacelike, null, and timelike geodesics, respectively. Since we consider the null geodesics, we choose $\delta=0$ here. Then the radial motion can be expressed as

$$
\dot{r}^{2}+\mathscr{V}_{\text {eff }}(r)=E^{2}
$$

with the effective potential $\mathscr{V}_{\text {eff }}=\left(L^{2} / r^{2}\right)\left(1-\left(2 M r^{2} /\left(r^{3}+\right.\right.\right.$ $\left.\left.2 q^{2} M\right)\right)$ ). Then the circular geodesics satisfy

$$
\mathscr{V}_{\text {eff }}(r)=E^{2}, \quad \frac{\partial \mathscr{V}_{\text {eff }}}{\partial r}=0 .
$$

Moreover, a stable (unstable) circular orbit requires $\partial^{2} \mathscr{V}_{\text {eff }} /$ $\partial r^{2}>0(<0)$, which admits a minimum (maximum) of the effective potential. Solving (12), we have

$$
2 f(r)-r f^{\prime}(r)=0 .
$$

And, for the metric (2), this equation reduces to

$$
2 r^{6}-3 r^{5}+4 q^{2} r^{3}+2 q^{4}=0,
$$

where, for simplicity, we measure all quantities with the Schwarzschild radius, which is equivalent to putting $2 M=1$ in all equations. Solving (14), we can obtain the stable and unstable circular orbits for this nonsingular spacetime. For a spherically symmetric and static spacetime, the photon sphere is known as an unstable circular orbit of photon (other definitions can be found in $[25,65])$. So, we can obtain the photon sphere for this nonsingular spacetime from (14) with the unstable condition $\partial^{2} \mathscr{V}_{\text {eff }} / \partial r^{2}<0$. It is obvious that this relation (14) is quite different from that in the Schwarzschild black hole spacetime, which implies that, in the strong field limit, there exist some distinct effects of $q$ on the gravitational lensing. The stable circular orbit can also be got by imposing $\partial^{2} \mathscr{V}_{\text {eff }} / \partial r^{2}>0$. The event horizons, photon sphere, and stable circular orbit are plotted in Figure 1 as a function of $q / 2 M$. It is clear that there are several distinct ranges of the parameter emerging where the structures of the horizons and circular geodesics will be qualitatively different; namely, $q / 2 M \epsilon$ $\left(0, Q_{\mathrm{cr} 1}\right), q / 2 M=Q_{\mathrm{cr} 1}, q / 2 M \in\left(Q_{\mathrm{cr} 1}, Q_{\mathrm{cr} 2}\right), q / 2 M=Q_{\mathrm{cr} 2}$, and $q / 2 M \in\left(Q_{\mathrm{cr} 2}, \infty\right)$. In the following we will discuss these cases, respectively.

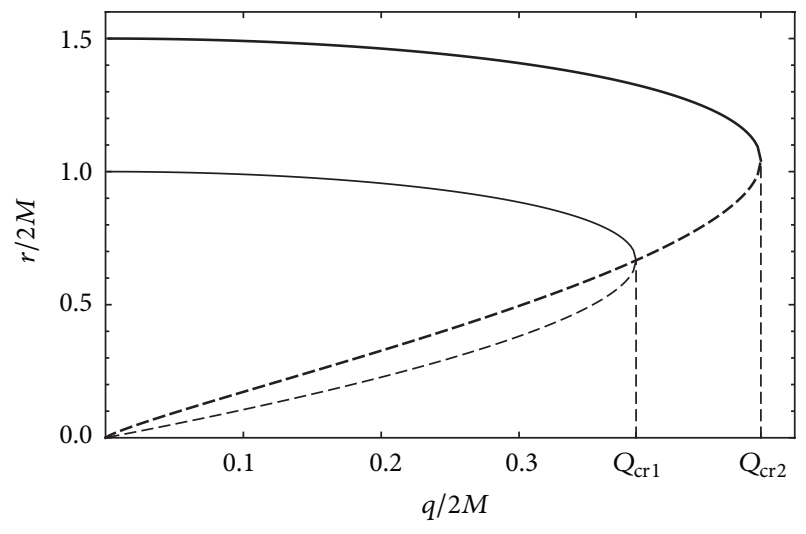

FIGURE 1: Horizons and circular orbits for the nonsingular spacetime. The thin solid and dashed lines are for the outer and inner horizons, respectively. And the thick solid and dashed lines are for the photon sphere and stable circular orbit, respectively. The parameters $Q_{\mathrm{cr} 1}=(2 \sqrt{3}) / 9=0.385$ and $Q_{\mathrm{cr} 2}=(25 \sqrt{30}) / 288=$ 0.475 .

Case 1 (nonsingular black hole $q / 2 M \in\left(0, Q_{\mathrm{cr} 1}\right)$ ). Since $\mathscr{V}_{\text {eff }} \sim f(r)$, the horizon locates at $\mathscr{V}_{\text {eff }}=0$. And note that the local minimum and maximum of $\mathscr{V}$ eff correspond to the stable and unstable circular orbits, respectively. Then we can easily read the property of different cases from Figure 2.

For the case of a nonsingular black hole with $q / 2 M \epsilon$ $\left(0, Q_{\mathrm{crl}}\right)$, there are two horizons $r_{ \pm}$, one photon sphere $r_{\mathrm{ps}}$, and one stable circular orbit $r_{\text {cir }}$. And they satisfy the following relation:

$$
r_{-}<r_{\mathrm{cir}}<r_{+}<r_{\mathrm{ps}}
$$

This implies that the outer horizon is always covered by a photon sphere, and the stable circular orbit locates between these two horizons.

In Figure 2(a), the general behavior of the effective potential $\mathscr{V}_{\text {eff }}(r)$ is shown as a function of $r$ for different values of the angular momentum $L$. We find that the effective potential admits two zeros corresponding to the outer and inner horizons, as well as one maximum and one minimum corresponding to the photon sphere and stable circular orbit. We can also see that the local minimum point always lies between these two zeros indicating the stable circular orbit lies in the region between the two horizons.

Case 2 (extremal nonsingular black hole $q / 2 M=Q_{\mathrm{crl}}$ ). For this case, we have the following relation:

$$
r_{-}=r_{+}=r_{\text {cir }}<r_{\mathrm{ps}} .
$$

The first "=" means that the two horizons coincide with each other. This case corresponds to an extremal nonsingular black hole. The second "=" implies that the degenerate horizon is also a stable circular orbit against small perturbation.

The detailed behavior of the effective potential $\mathscr{V}_{\text {eff }}(r)$ is presented in Figure 2(b). It shows that the effective potential has one zero and one minimum located at the same point. It also admits a maximum corresponding to the photon sphere. 


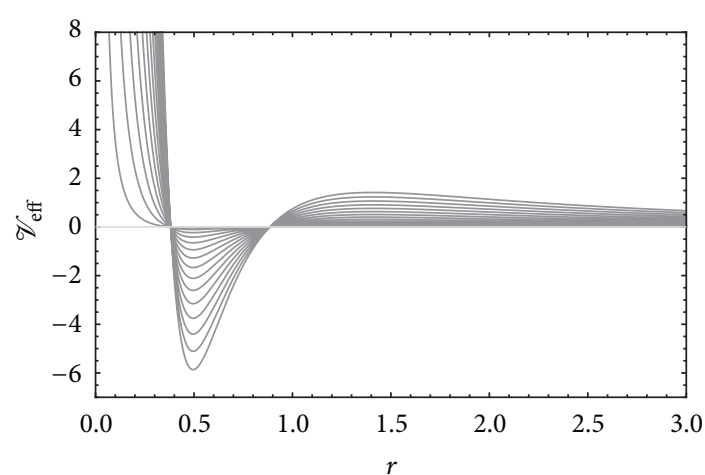

(a) The nonsingular black hole

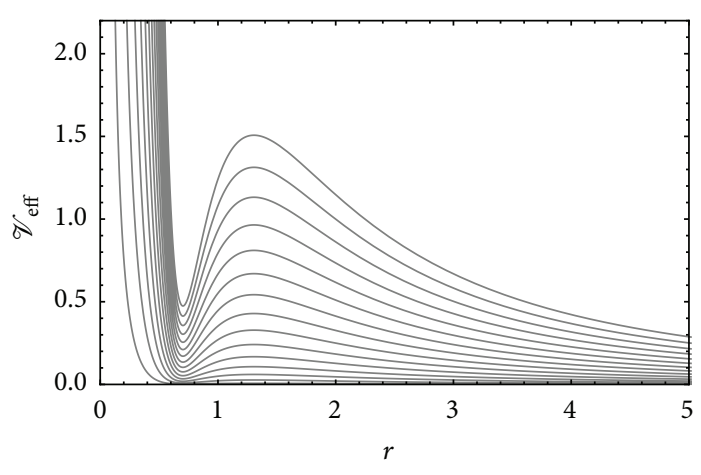

(c) The weakly nonsingular spacetime

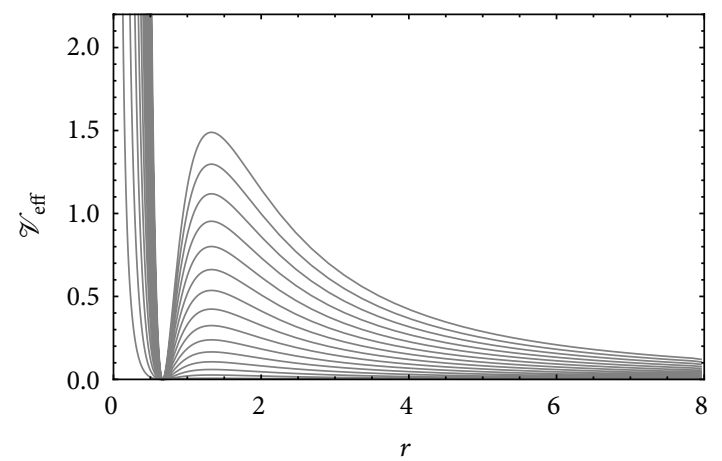

(b) The extremal nonsingular black hole

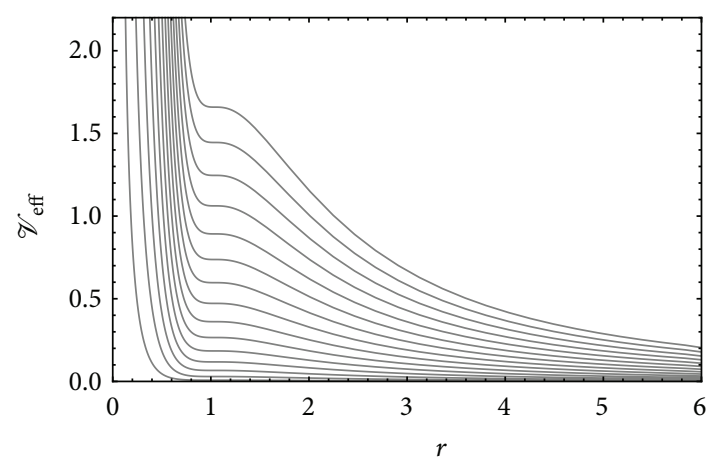

(d) The marginally nonsingular spacetime

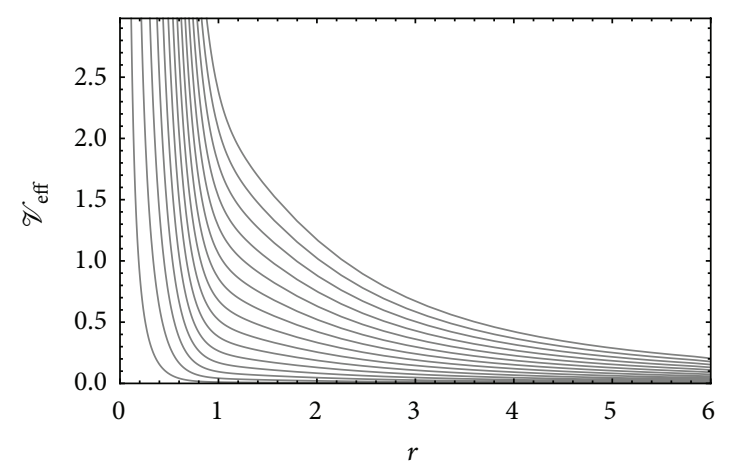

(e) The strongly nonsingular spacetime

FIgURE 2: Behavior of the effective potential $\mathscr{V}_{\text {eff }}$ as a function of $r$ with different values of $L$.

Case 3 (weakly nonsingular spacetime $q / 2 M \in\left(Q_{\mathrm{cr} 1}, Q_{\mathrm{cr} 2}\right)$ ). For the case of $q / 2 M \in\left(Q_{\mathrm{cr} 1}, Q_{\mathrm{cr} 2}\right)$, we clearly see from Figure 1 that the horizon disappears. So this case describes a nonsingular spacetime without a black hole. Obviously, the radius of the photon sphere and stable circular orbit satisfy

$$
r_{\text {cir }}<r_{\text {ps }} .
$$

This tells that the stable circular orbit is always covered by a photon sphere. The effective potential $\mathscr{V}_{\text {eff }}(r)$ is plotted in Figure 2(c), from which we find that $\mathscr{V}_{\text {eff }}(r)$ is positive for all $r$ 's. Thus, the horizons do not exist in this case, which is consistent with the result from Figure 1. On the other hand, there are one minimum and one maximum corresponding to the stable circular orbit and photon sphere, respectively.
Case 4 (marginally nonsingular spacetime $q / 2 M=Q_{\mathrm{cr} 2}$ ). For this case, the horizon also disappears. And the stable and unstable circular orbits coincide with each other; that is, $r_{\text {cir }}=r_{\mathrm{ps}}$. For this circular orbit, we have $\partial^{2} \mathscr{V}_{\text {eff }} / \partial r^{2}=0$. This result can be seen from Figure 2(d). One the other hand, we will see in the next section that some strong deflection limit coefficients will diverge in this case caused by $r_{\text {cir }}=r_{\mathrm{ps}}$.

Case 5 (strongly nonsingular spacetime $q / 2 M \in\left(Q_{\mathrm{cr} 2}, \infty\right)$ ). For the last case, all the horizons, photon sphere, and the stable circular orbit disappear. The effective potential $\mathscr{V}_{\text {eff }}(r)$ is plotted in Figure 2(e). It monotonically decreases from infinity to zero as $r$ goes from 0 to $\infty$.

From the above discussion, we find that the photon sphere of a nonsingular black hole is always larger than that 
of a weakly nonsingular spacetime. Therefore, we come to the conclusion that a photon is more easily captured by a nonsingular black hole. Since the photon sphere disappears for a strongly nonsingular spacetime, we will not focus on the lensing for a strongly nonsingular spacetime.

\section{Lensing in Nonsingular Spacetime}

In this section, we will study the lensing in nonsingular spacetime. The influence of nonsingularity parameter $q$ on the position and magnification of the nonrelativistic and relativistic images will be analyzed.

3.1. The Deflection Angle and Lens Geometry. Taking into account the spherical symmetry of this spacetime, we just consider the case that both the observer and the source lie in the equatorial plane $(\theta=\pi / 2)$, resulting in the fact that the whole trajectory of the photon is also restricted in the same plane. Then the deflection angle for the photon coming from infinity and returning to infinity is

$$
\alpha\left(r_{0}\right)=I\left(r_{0}\right)-\pi,
$$

with the total azimuthal angle given by

$$
I\left(r_{0}\right)=2 \int_{r_{0}}^{\infty} \frac{r_{0}}{r} \frac{d r}{\sqrt{r^{2} f\left(r_{0}\right)-r_{0}^{2} f(r)}},
$$

where (9) and (11) are used and $E$ is set to one. In a black hole spacetime, the deflection angle $\alpha\left(r_{0}\right)$ is a monotonically decreasing function with $r_{0}$. So it is easy to imagine that the light ray can make a complete loop or more than one loop before reaching the observer. As other authors have pointed out, the value of the deflection angle will be unboundedly large when the photon sphere is reached.

Defining $x=r_{0} / r$, the total azimuthal angle can be expressed as

$$
I\left(r_{0}\right)=\int_{0}^{1} h(x) d x
$$

with

$$
h(x)=-2 \sqrt{\frac{\left(q^{2}+r_{0}^{3}\right)\left(q^{2} x^{3}+r_{0}^{3}\right)}{\left(1-x^{2}\right)\left(q^{2}+r_{0}^{3}\right)\left(q^{2} x^{3}+r_{0}^{3}\right)+r_{0}^{5}\left(x^{3}-1\right)}}
$$

Here we would like to give a brief study on the lens geometry. The lens configuration is supposed in which the black hole is situated between the light source and observer; both of them are far from the black hole lens, so that the gravitational fields are very weak and the spacetime there can be described by a flat metric. An important element of the lens geometry is the optical axis, which is defined as the line joining the observer and the lens. The angular positions of the source (S) and the images (I), seen from the observer, are denoted by $\omega$ and $\theta$. Then the lens equation reads [25]

$$
\tan \omega=\tan \theta-\frac{D_{\mathrm{LS}}}{D_{\mathrm{OS}}}[\tan (\alpha-\theta)+\tan \theta] .
$$

$D_{\mathrm{OL}}, D_{\mathrm{LS}}$, and $D_{\mathrm{OS}}$ are the observer-lens, lens-source, and observer-source distances, respectively. It was pointed out by Bozza [66] that these distances are not the true distances between different positions; however, if the source is very close to the optical axis, they are a reasonably good approximation.

3.2. Nonrelativistic Images. Let us first consider the lensing in the weak deflection limit, where photon has a large impact parameter; that is, $r_{0} \gg 1$. Then the function $h(x)$ in (21) can perform a Taylor expansion around $1 / r_{0}$, which is given by

$$
\begin{aligned}
h(x)= & \frac{2}{\sqrt{1-x^{2}}}+\frac{x^{2}+x+1}{(x+1) \sqrt{1-x^{2}}} \frac{1}{r_{0}} \\
& +\frac{3\left(x^{2}+x+1\right)^{2}}{4(x+1)^{2} \sqrt{1-x^{2}}} \frac{1}{r_{0}^{2}} \\
& +\frac{5\left(x^{2}+x+1\right)^{3}}{8(x+1)^{3} \sqrt{1-x^{2}} \frac{1}{r_{0}^{3}}} \\
& +\frac{35\left(x^{2}+x+1\right)^{4}-64 q^{2}(x+1)^{4}\left(x^{4}+x^{2}+1\right)}{64(x+1)^{4} \sqrt{1-x^{2}}} \frac{1}{r_{0}^{4}} \\
& +\mathcal{O}\left(\frac{1}{r_{0}^{5}}\right) .
\end{aligned}
$$

Thus, the deflection angle is calculated as

$$
\alpha\left(r_{0}\right)=A_{1} \frac{1}{r_{0}}+A_{2} \frac{1}{r_{0}^{2}}+A_{3} \frac{1}{r_{0}^{3}}+A_{4} \frac{1}{r_{0}^{4}}+\mathcal{O}\left(\frac{1}{r_{0}^{5}}\right)
$$

with the coefficients

$$
\begin{gathered}
A_{1}=2, \quad A_{2}=\frac{15 \pi-16}{16}, \quad A_{3}=-\frac{45 \pi-244}{48}, \\
A_{4}=\left(2.94524 q^{2}-2.50549\right) .
\end{gathered}
$$

Note that the nonsingularity parameter $q$ only affects the deflection angle in the fourth order. Since $r_{0}$ has a large value, the influence of $q$ on the nonrelativistic images is very weak. Restoring the dimension, the first three coefficients are exactly consistent with that of [67].

For high alignment and using lens equation (22), the image positions and magnifications in the weak deflection limit can be written as a series expansion of the form [67]

$$
\begin{aligned}
& \theta=\theta_{0}+\theta_{1} \epsilon+\theta_{2} \epsilon^{2}+\theta_{3} \epsilon^{3}+O\left(\epsilon^{4}\right), \\
& \mu=\mu_{0}+\mu_{1} \epsilon+\mu_{2} \epsilon^{2}+\mu_{3} \epsilon^{3}+O\left(\epsilon^{4}\right),
\end{aligned}
$$

where the expansion parameter $\epsilon=\theta_{E} D_{\mathrm{OS}} / 4 D_{\mathrm{LS}}$ denotes the angle subtended by the gravitational radius normalized by the angular Einstein radius. It is easy to check that the coefficients $\theta_{0} \sim \theta_{2}$ and $\mu_{0} \sim \mu_{2}$ are independent of $q$, and their forms can be found in [67]. Thus, the nonsingularity parameter $q$ only 
affects the position and magnification of images of more than the third order in $\epsilon$. As a result, we come to the conclusion that the influence of the nonsingularity parameter can be ignored in the weak deflection limit.

3.3. Relativistic Images. In this case, the spacetime always has a photon sphere. And a photon before reaching the observer can do many loops around the black hole; therefore the photon should pass very near the photon sphere. Adopting the method developed by Bozza et al., we define a variable [31]

$$
z=\frac{f(r)-f\left(r_{0}\right)}{1-f\left(r_{0}\right)} .
$$

For the photon at infinity $r=\infty$, one has $z=1$ for $f(\infty)=$ 1. And when $r=r_{0}$, one easily gets $z=0$. Then the total azimuthal angle (20) can be rewritten as

$$
I\left(r_{0}\right)=\int_{0}^{1} R\left(z, r_{0}\right) K\left(z, r_{0}\right) d z
$$

where

$$
\begin{aligned}
R\left(z, r_{0}\right)= & \frac{2 r_{0}\left(1-f\left(r_{0}\right)\right)}{r^{2} f(r) f^{\prime}(r)}=\frac{2 r_{0}^{3}\left(r^{3}+q^{2}\right)^{2}}{r^{3}\left(r_{0}^{3}+q^{2}\right)\left(r^{3}-2 q^{2}\right)} \\
K\left(z, r_{0}\right) & =\frac{r}{\sqrt{r^{2} f\left(r_{0}\right)-f(r) r_{0}^{2}}} \\
& =\left(1+\frac{r_{0}^{2}}{r^{3}+q^{2}}-\frac{r_{0}^{2}}{r_{0}^{3}+q^{2}}-\frac{r_{0}^{2}}{r^{2}}\right)^{-1 / 2},
\end{aligned}
$$

with $r=f^{-1}\left(\left(1-f\left(r_{0}\right)\right) z+f\left(r_{0}\right)\right)$. Note that the function $R\left(z, r_{0}\right)$ is regular for $z$ and $r_{0}$, while $K\left(z, r_{0}\right)$ diverges at $z=0$. So, we split the integral (28) into two parts:

$$
I\left(r_{0}\right)=I_{R}\left(r_{0}\right)+I_{D}\left(r_{0}\right),
$$

where the regular and divergent parts $I_{R}\left(r_{0}\right), I_{D}\left(r_{0}\right)$ are, respectively, given by

$$
\begin{gathered}
I_{R}=\int_{0}^{1} g\left(z, r_{0}\right) d x, \\
I_{D}=\int_{0}^{1} R\left(0, r_{\mathrm{ps}}\right) K_{0}\left(z, r_{0}\right) d z,
\end{gathered}
$$

with $g\left(z, r_{0}\right)=R\left(0, r_{0}\right) K\left(z, r_{0}\right)-R\left(0, r_{\mathrm{ps}}\right) K_{0}\left(z, r_{0}\right)$. In order to find the divergence of the integrand, we do a Taylor expansion of the function inside the square root of $K\left(z, r_{0}\right)$ and obtain the function $K_{0}\left(z, r_{0}\right)$ :

$$
K_{0}\left(z, r_{0}\right)=\frac{1}{\sqrt{\chi\left(r_{0}\right) z+\xi\left(r_{0}\right) z^{2}+\mathcal{O}\left(z^{3}\right)}},
$$

where the coefficients $\chi\left(r_{0}\right)$ and $\xi\left(r_{0}\right)$ read

$$
\begin{gathered}
\chi\left(r_{0}\right)=2-\frac{r_{0}^{2}}{r_{0}^{3}+q^{2}}-2 \frac{r_{0}^{2}-3 q^{2}}{r_{0}^{3}-2 q^{2}}, \\
\xi\left(r_{0}\right)=-1+3 \frac{r_{0}^{8}-\left(6 r_{0}-1\right) q^{2} r_{0}^{5}+3 r_{0}^{3} q^{4}}{\left(r_{0}^{3}-2 q^{2}\right)^{3}} .
\end{gathered}
$$

We find that when $r_{0}$ approaches $r_{\mathrm{ps}}$, the coefficient $\chi\left(r_{0}\right)$ vanishes, and the leading term of the divergence in $H_{0}$ is $z^{-1}$, which would lead to the logarithmic divergence of the integrand. Thus, near $r_{\mathrm{ps}}$, the deflection angle can be assumed in the form

$$
\alpha(u)=-\bar{a} \log \left(\frac{u}{u_{\mathrm{ps}}}-1\right)+\bar{b}+\mathcal{O}\left(u-u_{\mathrm{ps}}\right) .
$$

Under this assumption, the minimum impact parameter $u_{\mathrm{ps}}$ and the strong deflection limit coefficients $\bar{a}$ and $\bar{b}$ are

$$
\begin{aligned}
& u_{\mathrm{ps}}=\left.\sqrt{\frac{r^{2}}{f(r)}}\right|_{r=r_{\mathrm{ps}}}=\sqrt{\frac{r_{\mathrm{ps}}^{3}+q^{2}}{r_{\mathrm{ps}}^{2}\left(r_{\mathrm{ps}}-1\right)+q^{2} / r_{\mathrm{ps}}^{2}}}, \\
& \bar{a}=\frac{R\left(0, r_{\mathrm{ps}}\right)}{2 \sqrt{\xi\left(r_{\mathrm{ps}}\right)}}=\sqrt{\frac{\left(r_{\mathrm{ps}}^{3}+q^{2}\right)\left(r_{\mathrm{ps}}^{3}-2 q^{2}\right)}{\left(3-r_{\mathrm{ps}}\right) r_{\mathrm{ps}}^{5}-11 q^{2} r_{\mathrm{ps}}^{3}+8 q^{4}}}, \\
& \bar{b}=-\pi+b_{R}+\bar{a} \log \kappa,
\end{aligned}
$$

where

$$
\kappa=\frac{2 \xi\left(r_{\mathrm{ps}}\right)}{f\left(r_{\mathrm{ps}}\right)}=\frac{2\left(r_{\mathrm{ps}}^{3}+q^{2}\right)^{2}\left(\left(3-r_{\mathrm{ps}}\right) r_{\mathrm{ps}}^{5}-11 q^{2} r_{\mathrm{ps}}^{3}+8 q^{4}\right)}{\left(r_{\mathrm{ps}}^{3}-r_{\mathrm{ps}}^{2}+q^{2}\right)\left(r_{\mathrm{ps}}^{3}-2 q^{2}\right)^{3}} .
$$

For this nonsingular spacetime, the coefficient $b_{R}$ cannot be calculated analytically. In order to obtain it, we expand $I_{R}\left(r_{0}\right)$ around $r_{\mathrm{ps}}$ :

$$
I_{R}\left(r_{0}\right)=\left.\sum_{n=0}^{\infty} \frac{1}{n !}\left(r_{0}-r_{\mathrm{ps}}\right)^{n} \int_{0}^{1} \frac{\partial^{n} g}{\partial r_{0}^{n}}\right|_{r_{0}=r_{\mathrm{ps}}} d z .
$$

Therefore, ignoring the higher-order terms, we get

$$
b_{R}=I_{R}\left(r_{\mathrm{ps}}\right)=\int_{0}^{1} g\left(z, r_{\mathrm{ps}}\right) d z .
$$

With this equation, we can numerically calculate $b_{R}$. It is worth pointing out that this result is accurate for the case $r_{0} \sim r_{\mathrm{ps}}$, while it is invalid for $r_{0} \gg r_{\mathrm{ps}}$. From these strong deflection limit coefficients, one easily sees that there is a significant effect of the nonsingularity parameter $q$ on the strong gravitational lensing. When $q=0$, these parameters will reduce to the case of the Schwarzschild black hole $[31,36]$.

The behaviors of $u_{\mathrm{ps}}, \bar{a}$, and $\bar{b}$ are presented in Figure 3. The result shows that the minimum impact parameter has a similar behavior as the radius of the photon sphere. We can also find that the strong deflection limit coefficient $\bar{a}$ grows with $q / 2 M$, while $\bar{b}$ decreases. For a nonsingular black hole, both $\bar{a}$ and $\bar{b}$ have a finite value. Compared with the result of nonsingular black hole, in the weakly nonsingular spacetime, it has small values of $u_{\mathrm{ps}}$ and $\bar{b}$, while it has large value of $\bar{a}$. In particular, $\bar{a}$ goes to positive infinity and $\bar{b}$ goes to negative infinity when the nonsingularity parameter 


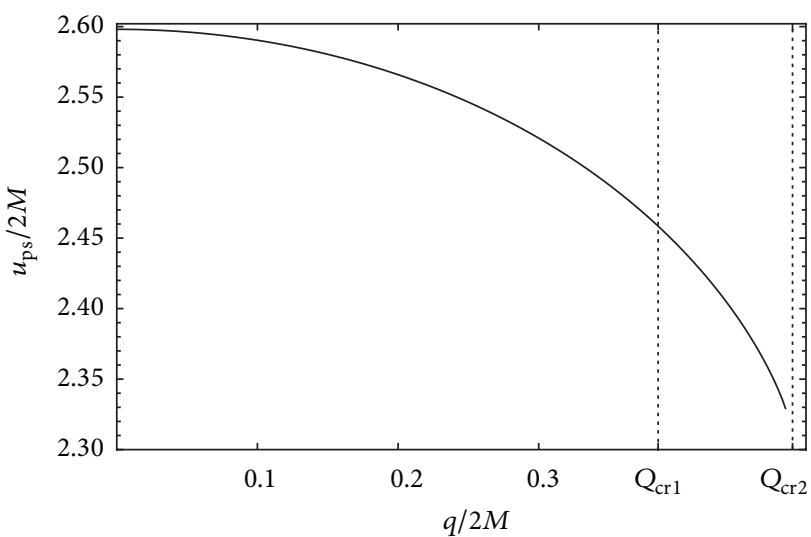

(a)

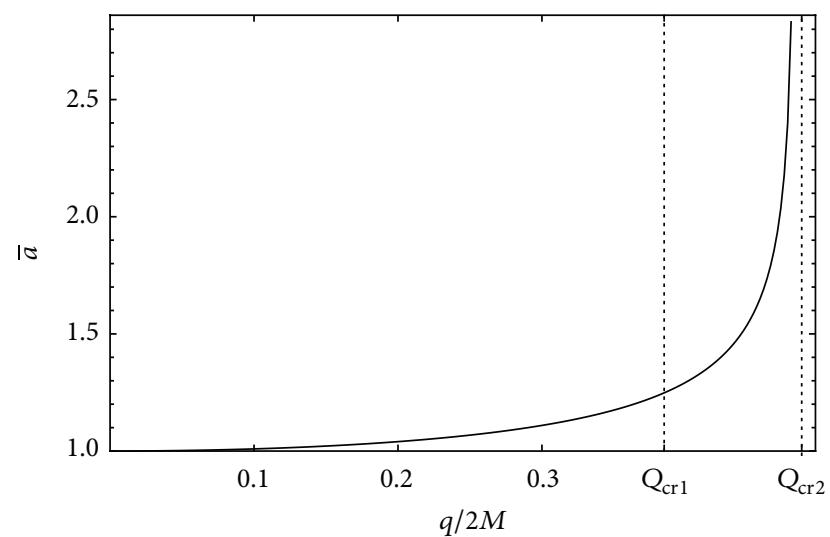

(b)

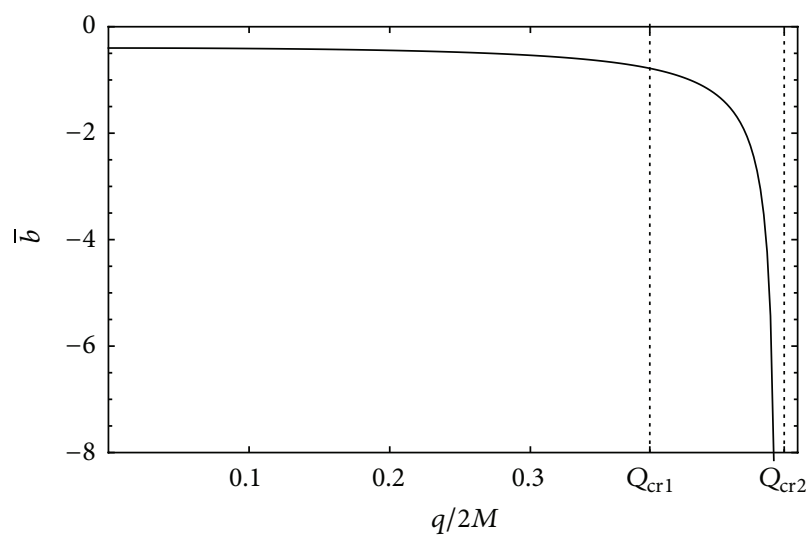

(c)

FIGURE 3: Behaviors of the minimal impact parameter $u_{\mathrm{ps}}$ and the strong deflection limit coefficients $\bar{a}$ and $\bar{b}$.

$q / 2 M$ approaches $Q_{\mathrm{cr} 2}$, where the marginally nonsingular spacetime arrives. With the values of these coefficients, one can obtain the behavior of the deflection angle. For fixed impact parameter $u$, the deflection angle increases with $q / 2 M$ shown in Figure 4(a). And, in Figure 4(b), it is shown that $\alpha$ decreases with $\left(u-u_{\mathrm{ps}}\right)$ for fixed $q / 2 M$. However, the angle will be unboundedly large when $u \rightarrow u_{\mathrm{ps}}$, which corresponds to the case that $r_{0}$ approaches $r_{\mathrm{ps}}$.

Here, we consider that the source, lens, and observer are highly aligned; that is, $\emptyset, \theta \ll 1$; lens equation (22) is reduced to $[31,36]$

$$
\varpi=\theta-\frac{D_{\mathrm{LS}}}{D_{\mathrm{OS}}} \Delta \alpha_{n}
$$

where $\Delta \alpha_{n}=\alpha-2 n \pi$ and $n$ denotes the number of the loops that the photon did around the lens. Using the lens geometry, we can obtain the angular position and magnification of the images [31, 36]:

$$
\begin{aligned}
& \theta_{n}=\theta_{n}^{0}+\frac{u_{\mathrm{ps}} e_{n}}{\bar{a}} \frac{D_{\mathrm{OS}}}{D_{\mathrm{OL}} D_{\mathrm{LS}}}\left(\omega-\theta_{n}^{0}\right), \\
& \mu_{n}=\frac{e_{n}\left(1+e_{n}\right)}{\bar{a} \omega} \frac{D_{\mathrm{OS}}}{D_{\mathrm{OL}}}\left(\frac{u_{\mathrm{ps}}}{D_{\mathrm{OL}}}\right)^{2},
\end{aligned}
$$

where

$$
\begin{gathered}
e_{n}=e^{(\bar{b}-2 n \pi) / \bar{a}}, \\
\theta_{n}^{0}=\frac{u_{\mathrm{ps}}}{D_{\mathrm{OL}}}\left(1+e_{n}\right) .
\end{gathered}
$$

Since the magnification $\mu_{n} \sim e^{-n}$, the first image is the brightest one. We can also see that $\mu_{n}$ is proportional to the small quantity $\left(u_{\mathrm{ps}} / D_{\mathrm{OL}}\right)^{2}$, which leads to faint images. Thus, we have the conclusion that, for nonzero $\omega$, the first image is the brightest one among these relativistic images and its brightness decreases quickly with the distance $D_{\mathrm{OL}}$. On the other hand, it is worth noting that when $\emptyset=0$, the magnification (41) will be no longer valid.

\section{Numerical Estimation of the Observables for the Supermassive Galactic Black Hole Lensing}

In this section, we first introduce three observables and then estimate the numerical values for the observables of gravitational lensing in the strong field limit. 


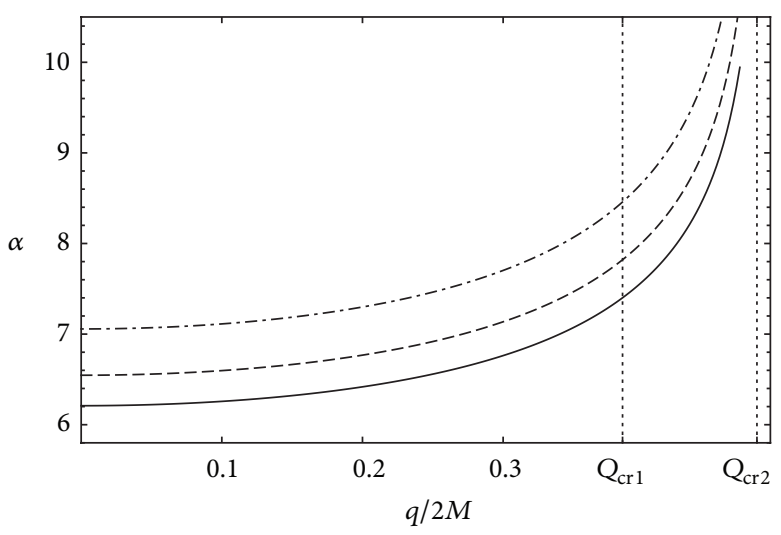

(a)

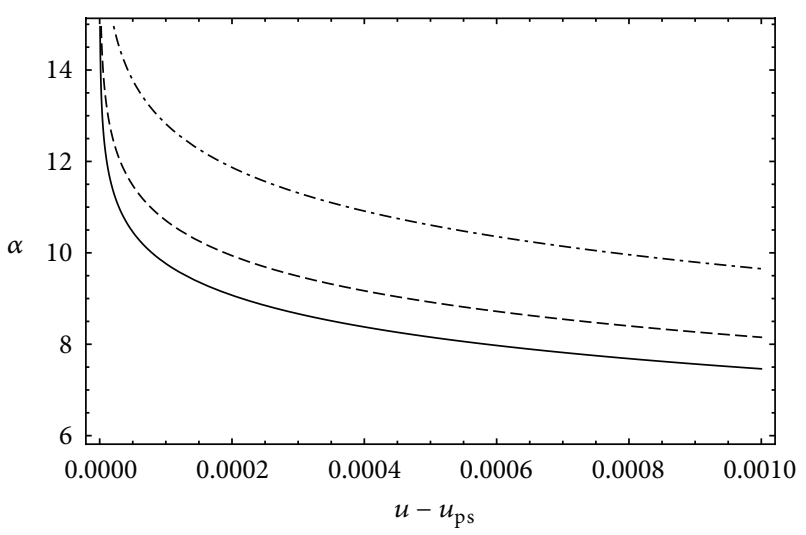

(b)

Figure 4: (a) The deflection angle $\alpha$ as a function of $q / 2 M$ with $u=u_{\mathrm{ps}}+0.0015, u_{\mathrm{ps}}+0.0025$, and $u_{\mathrm{ps}}+0.0035$ from the bottom to top. (b) The deflection angle $\alpha$ as a function of $\left(u-u_{\mathrm{ps}}\right)$ for $q / 2 M=0,0.3$, and 0.42 from the bottom to top.

Consider the case that the outermost image with angular position $\theta_{1}$ is a single image and the others are packed together at $\theta_{\infty}=u_{\mathrm{ps}} / D_{\mathrm{OL}}$. Then we have the observables [36]:

$$
\begin{gathered}
s=\theta_{1}-\theta_{\infty}=\theta_{\infty} e^{(\bar{b}-2 \pi) / \bar{a}}, \\
\tilde{r}=\frac{\mu_{1}}{\sum_{n=2}^{\infty} \mu_{n}}=e^{2 \pi / \bar{a}},
\end{gathered}
$$

where $s$ measures the angular separation between the first image and other ones and $\widetilde{r}$ denotes the flux of the first image and the sum of the others.

Next, we would like to estimate the numerical values for the observables of gravitational lensing in the strong field limit by supposing that the gravitational field of the supermassive black hole at the center of our Milky Way can be described by the nonsingular metric (1), and the gravitational field near the light source and the observer is very weak so that it can be described by a flat metric. The mass of the supermassive black hole and the distance between the observer and the black hole are estimated to be $M=2.8 \times$ $10^{6} M_{\odot}$ and $D_{\mathrm{OL}}=8.5 \mathrm{kpc}$ with $M_{\odot}$ being the mass of the sun. Under this assumption, the angular position can be calculated with the relation

$$
\theta_{\infty} \approx 1.97116 \times 10^{-5} u_{\mathrm{ps}}\left(\frac{M}{M_{\odot}}\right)\left(\frac{1 \mathrm{kpc}}{D_{\mathrm{OL}}}\right) \mu \operatorname{arcsec} .
$$

From this equation, we get that the angular position $\theta_{\infty}$ depends on the parameter $u_{\mathrm{ps}}$ and mass $M$ determined by the nature of the gravitational lens and also on the observerlens distance $D_{\mathrm{OL}}$ determined by the lens geometry. In order to obtain a large value of $\theta_{\infty}$ for a lens of the same type, it should have large mass and small $D_{\mathrm{OL}}$. For different values of the nonsingularity parameter $q$, the numerical values for the strong deflection limit coefficients and observables are listed in Table 1. It is clear that these results reduce to the Schwarzschild black hole spacetime when $q=0$. Moreover, we found that as $q$ increases, the angular position of the relativistic images $\theta_{\infty}$ and the relative magnitudes $r_{m}$ decrease, while the angular separation $s$ increases.
The behaviors of the observables can also be found in Figure 5. We can find that, in the weakly nonsingular spacetime, the angular position $s$ of the relativistic images $\theta_{\infty}$ and the relative magnitudes $r_{m}$ decrease more quickly, and the angular separation $s$ increases more rapidly than the case of the nonsingular black hole. It is also clear that the angular position has a maximum value $s=0.1235 \mu$ arcsec at $q / 2 M=0.4659$. Compared with a Schwarzschild black hole or a nonsingular black hole, a weakly nonsingular spacetime has a smaller angular position of the relativistic images and relative magnification of the outermost relativistic image with the other relativistic images. However, it has a larger angular separation for these relativistic images. From Figure 5(a), we find that the numerical value for the angular position of the innermost relativistic images $\theta_{\infty}$ of a nonsingular spacetime is of about $15.3 \sim 16.8 \mu$ arcsec. In principle, such a resolution is reachable by very long baseline interferometry (VLBI) projects and advanced radio interferometry between space and Earth (ARISE), which have the angular resolution of $10 \sim 100 \mu$ arcsec in the near infrared $[68,69]$. Therefore we are hopeful to observe these relativistic images within a not so far future.

\section{Summary}

In this paper, we have shown that, in the weak deflection limit, the influence of the nonsingularity parameter $q$ on the gravitational lensing is negligible. However, in the strong deflection limit, $q$ has a significant effect, which may offer a potentially powerful tool to probe the nonsingularity of spacetime.

First, we investigated the null geodesics of a nonsingular spacetime. According to the nature of the nonsingular spacetime, it is classified into several cases, such as the nonsingular black hole, the extremal nonsingular black hole, the weakly nonsingular spacetime, the marginally nonsingular spacetime, and the strongly nonsingular spacetime. We found that the photon sphere can exist not only in a nonsingular black hole background but also in a spacetime without a black hole. The result also shows that the photon is more easily 
TABLE 1: Numerical estimation for main observables and the strong field limit coefficients for a Schwarzschild black hole, a nonsingular black hole, and a weakly nonsingular spacetime, which is supposed to describe the object at the center of our galaxy; $r_{m}=2.5 \log \tilde{r}$.

\begin{tabular}{lcccccc}
\hline & $q / 2 M=0$ & $q / 2 M=0.2$ & $q / 2 M=0.3$ & $q / 2 M=0.39$ & $q / 2 M=0.42$ & $q / 2 M=0.45$ \\
\hline $\bar{a}$ & 1.0000 & 1.0402 & 1.1094 & 1.2625 & 1.3771 & 1.6277 \\
$\bar{b}$ & -0.4002 & -0.4446 & -0.5369 & -0.8112 & -1.0782 & -1.8353 \\
$u_{\mathrm{ps}} / R_{s}$ & 2.5981 & 2.5659 & 2.5209 & 2.4537 & 2.4217 & 2.3806 \\
$s(\mu$ arcsec $)$ & 0.0211 & 0.0259 & 0.0350 & 0.0578 & 0.0750 & 0.1055 \\
$r_{m}$ (magnitude $)$ & 6.8219 & 6.5583 & 6.1490 & 5.4036 & 4.9539 & 4.1911 \\
$\theta_{\infty}(\mu$ arcsec $)$ & 16.8699 & 16.6610 & 16.3688 & 15.9321 & 15.7243 & 15.4580 \\
\hline
\end{tabular}

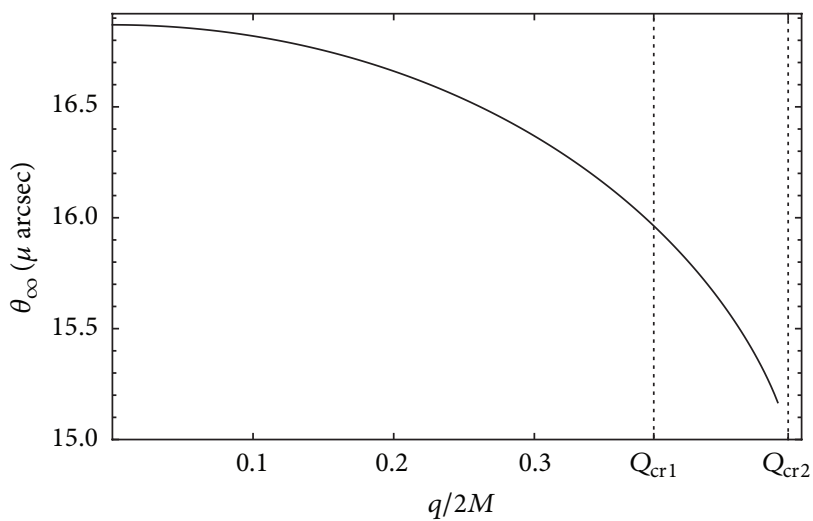

(a)

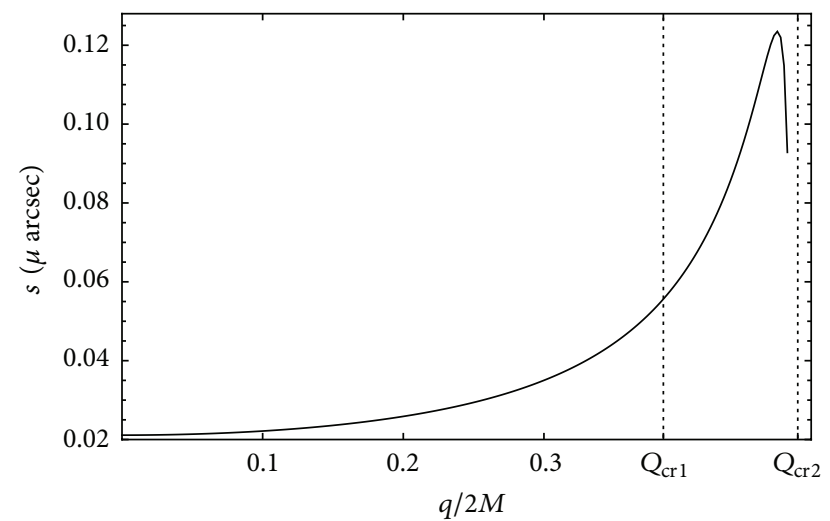

(b)

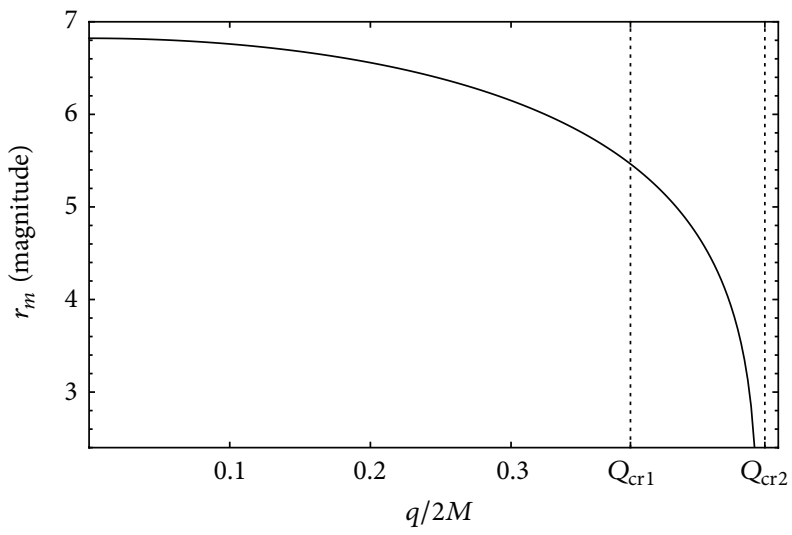

(c)

FIGURE 5: Gravitational lensing by the galactic center black hole. Variation of the values of the angular position $\theta_{\infty}$, angular separation $s$, and relative magnitudes $r_{m}$ with the parameter $q / 2 M$ in the nonsingular spacetime; $r_{m}=2.5 \log \widetilde{r}$.

captured by a nonsingular black hole rather than by a weakly nonsingular spacetime.

Second, based on the null geodesics, lensing in the weak and strong deflection limits was studied. For the first case, we found that the influence of the nonsingularity parameter $q$ on the positions and magnifications of the images is negligible. Thus, we cannot distinguish a nonsingular black hole from a Schwarzschild one. In the strong deflection limit, these strong deflection limit coefficients were also obtained. For a nonsingular black hole, these coefficients are always finite for any value of the nonsingularity parameter $q$. Compared with the result of nonsingular black hole, in the weakly nonsingular spacetime, it has small values of $u_{\mathrm{ps}}$ and $\bar{b}$, while it has large value of $\bar{a}$. And when the nonsingular spacetime approaches the marginally nonsingular one with $r_{\text {cir }}=r_{\text {ps }}$, we found that $\bar{a}$ goes to positive infinity and $\bar{b}$ goes to negative infinity. These results tell that the gravitational lensing by a weakly nonsingular spacetime is more obvious than a nonsingular black hole.

The model was also applied to the supermassive black hole hosted in the center of our Milky Way. It was shown that, with the increase of the nonsingularity parameter $q / 2 M$, the angular position of the relativistic images $\theta_{\infty}$ and the relative magnitudes $r_{m}$ decrease, while the angular separation $s$ 
increases. We found that, in a weakly nonsingular spacetime, the angular position of the relativistic images $\theta_{\infty}$ and relative magnitudes $r_{m}$ decrease more quickly, and the angular separation $s$ increases more rapidly than a nonsingular black hole.

As pointed out in [27], the relative magnitude $r_{m}$ obtained in this paper may have a large difference from its accurate value. However, the angular position $\theta_{\infty}$ and the angular separation $s$ are accurate enough. Thus, combined with the expected data from VLBI and ARISE, the strong gravitational lensing may offer a possible way to distinguish a weakly nonsingular spacetime from a nonsingular black hole. Furthermore, we are also allowed to probe the spacetime nonsingularity parameter $q$ by the astronomical instruments in the near future astronomical observations.

\section{Conflict of Interests}

The authors declare that there is no conflict of interests regarding the publication of this paper.

\section{Acknowledgments}

The authors would like to thank the anonymous referees whose comments largely helped them in improving the original paper. Shao-Wen Wei wishes to thank Dr. Changqing Liu for useful discussions and valuable comments. This work was supported by the National Natural Science Foundation of China (Grants nos. 11205074, 11075065, and 11375075) and the Fundamental Research Funds for the Central Universities (Grants nos. lzujbky-2013-21 and lzujbky-2013-18).

\section{References}

[1] R. Penrose, "Gravitational collapse and space-time singularities," Physical Review Letters, vol. 14, pp. 57-59, 1965.

[2] S. W. Hawking and R. Penrose, "The singularities of gravitational collapse and cosmology," Proceedings of the Royal Society of London. Series A: Mathematical, Physical and Engineering Sciences, vol. 314, no. 1519, pp. 529-548, 1970.

[3] S. W. Hawking, "Breakdown of predictability in gravitational collapse," Physical Review D, vol. 14, no. 10, pp. 2460-2473, 1976.

[4] P. Nicolini, A. Smailagic, and E. Spallucci, "The fate of radiating black holes in noncommutative geometry," ESA Special Publication, vol. 637, 11.1, 2006, http://arxiv.org/abs/hep-th/0507226.

[5] P. Nicolini, A. Smailagic, and E. Spallucci, "Noncommutative geometry inspired Schwarzschild black hole," Physics Letters B, vol. 632, no. 4, pp. 547-551, 2006.

[6] J. Bardeen, in Proccedings of GR5, USSR, Tbilisi, Georgia, 1968.

[7] A. Borde, "Open and closed universes, initial singularities, and inflation," Physical Review D, vol. 50, no. 6, pp. 3692-3702, 1994.

[8] A. Borde, "Regular black holes and topology change," Physical Review D, vol. 55, no. 12, pp. 7615-7617, 1997.

[9] E. Ayón-Beato and A. Garcia, "The Bardeen model as a nonlinear magnetic monopole," Physics Letters B, vol. 493, no. 1-2, pp. 149-152, 2000.

[10] S. A. Hayward, "Formation and evaporation of nonsingular black holes," Physical Review Letters, vol. 96, Article ID 031103, 2006.
[11] E. Poisson and W. Israel, "Structure of the black hole nucleus," Classical and Quantum Gravity, vol. 5, no. 12, pp. L201-L205, 1988.

[12] R. Balbinot and E. Poisson, "Stability of the Schwarzschild-de Sitter model," Physical Review D, vol. 41, no. 2, pp. 395-402, 1990.

[13] E. Spallucci and A. Smailagic, "Black holes production in selfcomplete quantum gravity," Physics Letters B, vol. 709, no. 3, pp. 266-269, 2012.

[14] A. Flachi and J. P. S. Lemos, "Quasinormal modes of regular black holes," Physical Review D, vol. 87, no. 2, Article ID 024034, 2013.

[15] M. Cataldo and A. Garcia, "Regular $(2+1)$-dimensional black holes within nonlinear electrodynamics," Physical Review D, vol. 61, Article ID 084003, 2000.

[16] A. Burinskii and S. R. Hildebrandt, "New type of regular black holes and particlelike solutions from nonlinear electrodynamics," Physical Review D, vol. 65, no. 10, Article ID 104017, 2002.

[17] K. A. Bronnikov and J. C. Fabris, "Regular phantom black holes," Physical Review Letters, vol. 96, Article ID 251101, 2006.

[18] W. Berej, J. Matyjasek, D. Tryniecki, and M. Woronowicz, "Regular black holes in quadratic gravity," General Relativity and Gravitation, vol. 38, no. 5, pp. 885-906, 2006.

[19] I. Dymnikova, "Regular electrically charged structures in nonlinear electrodynamics coupled to general relativity," Classical and Quantum Gravity, vol. 21, pp. 4417-4429, 2004.

[20] E. Ayón-Beato and A. García, "Regular black hole in general relativity coupled to nonlinear electrodynamics," Physical Review Letters, vol. 80, no. 23, pp. 5056-5059, 1998.

[21] J. P. S. Lemos and V. T. Zanchin, "Regular black holes: electrically charged solutions, Reissner-Nordström outside a de Sitter core," Physical Review D, vol. 83, no. 12, Article ID 124005, 2011.

[22] S. Ansoldi, "Spherical black holes with regular center: a review of existing models including a recent realization with Gaussian sources," Proceedings of the BH2, Dynamics and Thermodynamics of Black holes and Naked Singularities, http://arxiv.org/abs/0802.0330.

[23] C. Darwin, "The gravity field of a particle," Proceedings of the Royal Society. London. Series A. Mathematical, Physical and Engineering Sciences, vol. 249, pp. 180-194, 1959.

[24] K. S. Virbhadra, D. Narasimha, and S. M. Chitre, "Role of the scalar field in gravitational lensing," Astronomy \& Astrophysics, vol. 337, no. 1, pp. 1-8, 1998.

[25] K. S. Virbhadra and G. F. R. Ellis, "Schwarzschild black hole lensing," Physical Review D, vol. 62, no. 8, Article ID 084003, 2000.

[26] K. S. Virbhadra and G. F. R. Ellis, "Gravitational lensing by naked singularities," Physical Review D, vol. 65, no. 10, Article ID 103004, 2002.

[27] K. S. Virbhadra, "Relativistic images of Schwarzschild black hole lensing," Physical Review D, vol. 79, no. 8, Article ID 083004, 2009.

[28] E. F. Eiroa, G. E. Romero, and D. F. Torres, “Reissner-Nordström black hole lensing," Physical Review D, vol. 66, no. 2, 2002.

[29] V. Perlick, "Exact gravitational lens equation in spherically symmetric and static spacetimes," Physical Review D, vol. 69, no. 6, Article ID 064017, 2004.

[30] S. Frittelli, T. P. Kling, and E. T. Newman, "Spacetime perspective of Schwarzschild lensing," Physical Review D, vol. 61, Article ID 064021, 2000. 
[31] V. Bozza, S. Capozziello, G. Iovane, and G. Scarpetta, "Strong field limit of black hole gravitational lensing," General Relativity and Gravitation, vol. 33, no. 9, pp. 1535-1548, 2001.

[32] V. Bozza, "Quasi-equatorial gravitational lensing by spinning black holes in the strong field limit," Physical Review D, vol. 67, Article ID 103006, 2003.

[33] V. Bozza, F. de Luca, G. Scarpetta, and M. Sereno, "Analytic Kerr black hole lensing for equatorial observers in the strong deflection limit," Physical Review D, vol. 72, no. 8, Article ID 083003, 2005.

[34] V. Bozza, F. de Luca, and G. Scarpetta, "Kerr black hole lensing for generic observers in the strong deflection limit," Physical Review D, vol. 74, no. 6, Article ID 063001, 2006.

[35] V. Bozza, "Gravitational lensing by black holes," General Relativity and Gravitation, vol. 42, no. 9, pp. 2269-2300, 2010.

[36] V. Bozza, "Gravitational lensing in the strong field limit," Physical Review D, vol. 66, Article ID 103001, 2002.

[37] E. F. Eiroa, "Gravitational lensing by Einstein-Born-Infeld black holes," Physical Review D, vol. 73, no. 4, Article ID 043002, 2006.

[38] K. Sarkar and A. Bhadra, "Strong field gravitational lensing in scalar-tensor theories," Classical and Quantum Gravity, vol. 23, no. 22, pp. 6101-6113, 2006.

[39] S. Chen and J. Jing, "Strong field gravitational lensing in the deformed Hrava-Lifshitz black hole," Physical Review D, vol. 80, no. 2, Article ID 024036, 2009.

[40] E. F. Eiroa, G. E. Romero, and D. F. Torres, "Reissner-Nordstrom black hole lensing," Physical Review D, vol. 66, no. 2, Article ID 024010, 2002.

[41] E. F. Eiroa and C. M. Sendra, "Gravitational lensing by massless braneworld black holes," Physical Review D, vol. 86, no. 8, Article ID 083009, 2012.

[42] E. F. Eiroa and C. M. Sendra, "Regular phantom black hole gravitational lensing," Physical Review D, vol. 88, no. 10, Article ID 103007, 2013.

[43] R. Whisker, "Strong gravitational lensing by braneworld black holes," Physical Review D, vol. 71, Article ID 064004, 2005.

[44] A. Bhadra, "Gravitational lensing by a charged black hole of string theory," Physical Review D, vol. 67, no. 10, Article ID 103009, 2003.

[45] S. E. Vázquez and E. P. Esteban, "Strong-field gravitational lensing by a Kerr black hole," Il Nuovo Cimento della Società Italiana di Fisisca B, vol. 119, no. 5, pp. 489-519, 2004.

[46] K. K. Nandi, Y. Z. Zhang, and A. V. Zakharov, "Gravitational lensing by wormholes," Physical Review D, vol. 74, Article ID 024020, 2006.

[47] G. N. Gyulchev and S. S. Yazadjiev, "Kerr-Sen dilaton-axion black hole lensing in the strong deflection limit," Physical Review $D$, vol. 75, no. 2, Article ID 023006, 2007.

[48] G. N. Gyulchev and I. Z. Stefanov, "Gravitational lensing by phantom black holes," Physical Review D, vol. 87, no. 6, Article ID 063005, 2013.

[49] V. Bozza and G. Scarpetta, "Strong deflection limit of black hole gravitational lensing with arbitrary source distances," Physical Review D, vol. 76, no. 8, Article ID 083008, 2007.

[50] K. S. Virbhadra and C. R. Keeton, "Time delay and magnification centroid due to gravitational lensing by black holes and naked singularities," Physical Review D, vol. 77, no. 12, Article ID 124014, 2008.

[51] G. S. Bisnovatyi-Kogan and O. Y. Tsupko, "Strong gravitational lensing by Schwarzschild black holes," Astrophysics, vol. 51, no. 1, pp. 99-111, 2008.
[52] A. Y. Bin-Nun, "Strong gravitational lensing by Sgr A*," Classical and Quantum Gravity, vol. 28, no. 11, Article ID 114003, 2011.

[53] Y. Liu, S. Chen, and J. Jing, "Strong gravitational lensing in a squashed Kaluza-Klein black hole spacetime," Physical Review D, vol. 81, no. 12, Article ID 124017, 2010.

[54] C. Ding, S. Kang, C.-Y. Chen, S. Chen, and J. Jing, "Strong gravitational lensing in a noncommutative black-hole spacetime," Physical Review D, vol. 83, no. 8, Article ID 084005, 2011.

[55] C. Ding and J. Jing, "Probing spacetime noncommutative constant via charged astrophysical black hole lensing," Journal of High Energy Physics, vol. 2011, no. 10, article 52, 2011.

[56] C. Liu, S. Chen, and J. Jing, "Strong gravitational lensing of quasi-Kerr compact object with arbitrary quadrupole moments," Journal of High Energy Physics, vol. 2012, p. 97, 2012.

[57] T. Ghosh and S. SenGupta, "Strong gravitational lensing across a dilaton anti-de Sitter black hole," Physical Review D, vol. 81, no. 4, Article ID 044013, 2010.

[58] S. W. Wei and Y. X. Liu, "Equatorial and quasi-equatorial gravitational lensing by Kerr black hole pierced by a cosmic string," Physical Review D, vol. 85, Article ID 064044, 2012.

[59] S. W. Wei, Y. X. Liu, C.-E. Fu, and K. Yang, "Strong field limit analysis of gravitational lensing in Kerr-Taub-NUT spacetime," Journal of Cosmology and Astroparticle Physics, vol. 2012, no. 10, article 53, 2012.

[60] S. Sahu, M. Patil, D. Narasimha, and P. S. Joshi, "Can strong gravitational lensing distinguish naked singularities from black holes?" Physical Review D-Particles, Fields, Gravitation and Cosmology, vol. 86, no. 6, Article ID 063010, 2012.

[61] V. Perlick, "Gravitational lensing from a spacetime perspective," Living Reviews in Relativity, vol. 7, p. 9, 2004.

[62] I. Z. Stefanov, S. S. Yazadjiev, and G. G. Gyulchev, "Connection between black-hole quasinormal modes and lensing in the strong deflection limit," Physical Review Letters, vol. 104, no. 25, Article ID 251103, 2010.

[63] S. W. Wei, Y. X. Liu, and H. Guo, "Relationship between highenergy absorption cross section and strong gravitational lensing for black hole," Physical Review D, vol. 84, Article ID 041501(R), 2011.

[64] S. Chandrasekhar, The Mathematical Theory of Black Holes, Oxford University Press, New York, NY, USA, 1983.

[65] C.-M. Claudel, K. S. Virbhadra, and G. F. R. Ellis, "The geometry of photon surfaces," Journal of Mathematical Physics, vol. 42, no. 2, pp. 818-838, 2001.

[66] V. Bozza, "Comparison of approximate gravitational lens equations and a proposal for an improved new one," Physical Review D-Particles, Fields, Gravitation and Cosmology, vol. 78, no. 10, Article ID 103005, 2008.

[67] C. R. Keeton and A. O. Petters, "Formalism for testing theories of gravity using lensing by compact objects: static, spherically symmetric case," Physical Review D, vol. 72, no. 10, Article ID 104006, 2005.

[68] A. Eckart, T. Bertram, N. Mouawad, T. Viehmann, C. Straubmeier, and J. Zuther, "Long range science perspectives for the VLTI," Astrophysics and Space Science, vol. 286, no. 1-2, pp. 269276, 2003.

[69] V. Bozza and L. Mancini, "Observing gravitational lensing effects by Sgr A* with gravity," Astrophysical Journal, vol. 753, no. 1, article 56, 2012. 

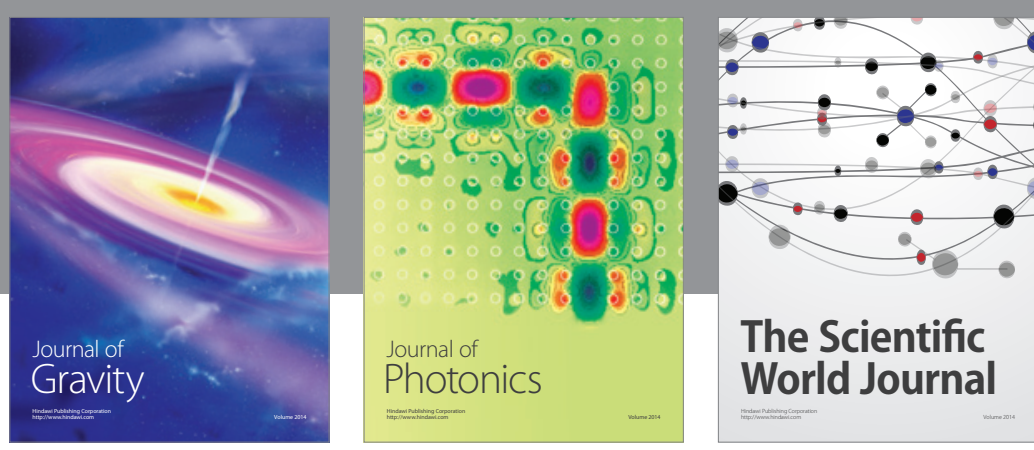

The Scientific World Journal
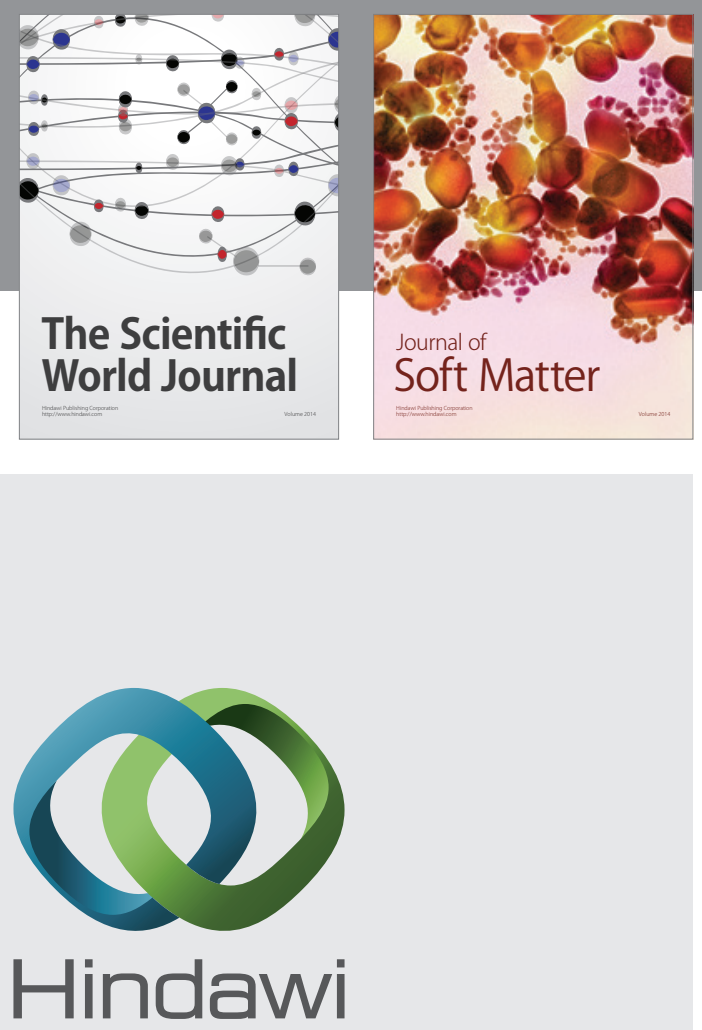

Submit your manuscripts at

http://www.hindawi.com

nternational Journal of

Statistical Mechanics
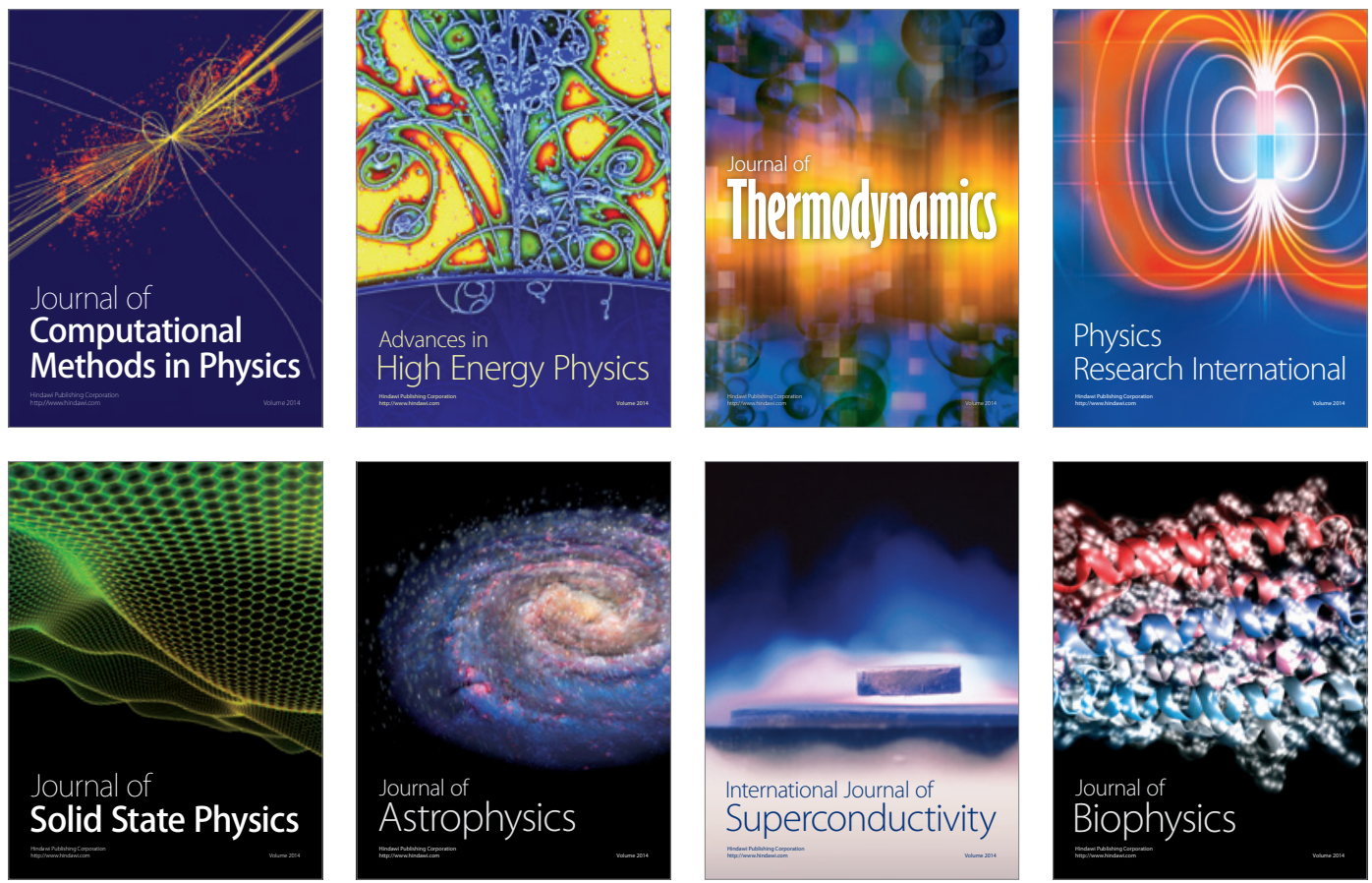
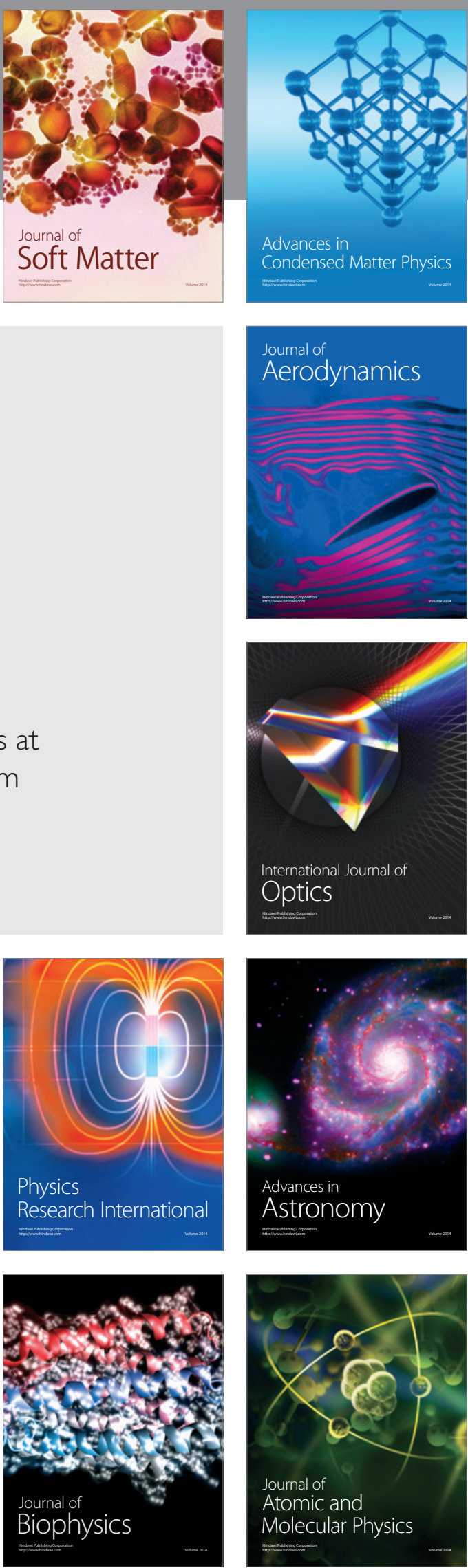